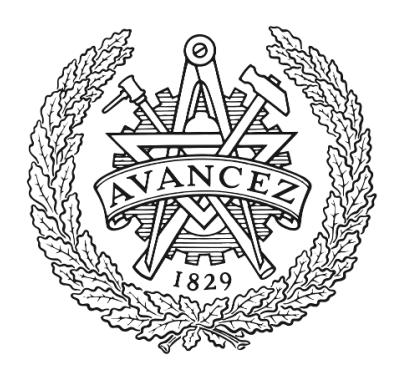

\title{
CHALMERS
}

UNIVERSITY OF TECHNOLOGY

\section{Delay-Accuracy Tradeoff in Opportunistic Time-of-Arrival Localization}

Downloaded from: https://research.chalmers.se, 2023-04-26 15:31 UTC

Citation for the original published paper (version of record):

Daniel, O., Wymeersch, H., Nurmi, J. (2018). Delay-Accuracy Tradeoff in Opportunistic

Time-of-Arrival Localization. IEEE Signal Processing Letters, 25(6): 763-767.

http://dx.doi.org/10.1109/LSP.2018.2826470

N.B. When citing this work, cite the original published paper.

C2018 IEEE. Personal use of this material is permitted.

However, permission to reprint/republish this material for advertising or promotional purposes 


\title{
Delay-Accuracy Trade-off in Opportunistic Time-of-Arrival Localization
}

\author{
Ondrej Daniel, Henk Wymeersch, Member, IEEE, and Jari Nurmi, Senior Member, IEEE
}

\begin{abstract}
While designing a positioning network, the localization performance is traditionally the main concern. However, collection of measurements together with channel access methods require a nonzero time, causing a delay experienced by network nodes. This fact is usually neglected in positioning-related literature. In terms of the delay-accuracy trade-off, broadcast schemes have an advantage over unicast, provided nodes can be properly synchronized. In this letter, we analyze the delay-accuracy tradeoff, for localization schemes in which the position estimates are obtained based on broadcasted ranging signals. We find that for dense networks, the trade-off is the same for cooperative and noncooperative networks, and cannot exceed a certain threshold value.
\end{abstract}

Index Terms-Localization, delays, accuracy, time division multiple access, broadcast.

\section{INTRODUCTION}

$\mathbf{I}$ $\mathrm{N}$ network localization, where nodes are divided into anchors (position references) and agents, performance is generally assessed in terms of the Fisher information, with networks that collect more measurements outperforming measurements with fewer measurements. In particular in cooperative localization, inter-agent measurements have been shown to be beneficial in terms of accuracy [1]-[3]. However, collecting more measurements comes at a cost in terms of delay [4], [5], requiring dedicated resource allocation strategies [6][8]. Under time-division multiple access (TDMA) and ultra wideband (UWB) ranging, [9] has characterized the trade-off between positioning accuracy and measurement delay, under various cooperative and noncooperative operating conditions, including unicast (relying on two-way time-of-arrival (TWTOA) distance measurements), unicast with eavesdroppers, and time-difference of arrival (TDOA with synchronized anchors). The main outcome was that for sparse agent networks (characterized by more anchors than agents), TDOA offered the best trade-off, while for dense agent networks, cooperative and noncooperative modes offered the same trade-off, with the best strategy being TW-TOA with eavesdropping. However, broadcast schemes other than TDOA were not studied, thus leaving open the question of how to achieve the best possible trade-off in the dense agent regime.

This work was supported by EU FP7 Marie Curie Initial Training Network MULTI-POS (Multi-technology Positioning Professionals) under grant no. 316528 and by the European Research Council under grant no. 258418 (COOPNET)

O. Daniel and J. Nurmi are with the Laboratory of Electronics and Communications Engineering Tampere University of Technology, 33720 Tampere, Finland (e-mail: ondrej.daniel@student.tut.fi, jari.nurm@tut.fi). H. Wymeersch is with the Department of Electrical Engineering, Chalmers University of Technology, 41296 Götenburg, Sweden (e-mail: henkw@ chalmers.se).
In this letter, motivated by the favorable trade-off values achieved by TDOA in the sparse agent regime, we focus on broadcast schemes for the dense agent regime. We consider schemes in which all the nodes (agents as well as anchors) may receive broadcasted ranging signals. Such signals may be sent by both agents and anchors (cooperative mode) and by anchors only (noncooperative mode). Since positioning is based on broadcast ranging signals (as opposed to the TW-TOA unicast approach), clock biases and transmission times must be incorporated into the measurement model and considered for estimation. As more parameters must be estimated, the trade-off may be affected. We establish the trade-off parameter for both cooperative and noncooperative networks. Our contributions are as follows: (i) we derive the Fisher information matrix for cooperative and noncooperative broadcast ranging; (ii) we demonstrate that, asymptotically, the equivalent Fisher information on the locations does not change under ideal synchronization; (iii) we establish that the optimal trade-off is bounded and the same for cooperative and noncooperative networks.

\section{SYSTEM MODEL}

We consider a complete network with $N$ agents and $M$ anchors located at positions $\mathbf{x}_{i}, i \in\{1, \ldots, M+N\}$. Nodes experience clock biases $o_{i}$ (assumed converted to meters) with the bias of the last node (arbitrarily) set to zero. Anchors have known locations, while agents do not. The main goal of the network is to determine the localization of the agents. We distinguish between cooperative (CO) and noncooperative (NC) network operating modes. In the former, all nodes broadcast and receive ranging signals, while in the latter, agents receive, but do not broadcast. In either case, a transmitting node $i$ broadcasts a ranging signal at time $t_{i}$. The signal is received by all other nodes. It leads to a measurement at a receiving node $j$ in the form of

$$
z_{i j}=\delta_{i}+d_{i j}+o_{j}+w_{i j}, \quad \text { for } i \neq j
$$

where $d_{i j}=\left\|\mathbf{x}_{i}-\mathbf{x}_{j}\right\|, w_{i j} \sim \mathcal{N}\left(0, \sigma^{2}\right)$, and $\delta_{i}=c \times t_{i}$ with $c$ being the signal propagation speed. The transmission times are unknown and determined through a rough packet-level synchronization. Only a single node is allowed to transmit at any given time (under TDMA). The aggregated measurement associated with the ranging transmissions from the node $i$ is denoted by $\mathbf{z}_{i}$, and we introduce $\mathbf{z}=\left[\mathbf{z}_{L}^{\mathrm{T}}, \ldots, \mathbf{z}_{M+N}^{\mathrm{T}}\right]^{\mathrm{T}}$, where $L$ allows to distinguish between the operating modes (i.e., $L=1$ for CO and $L=N+1$ for NC mode). The unknowns are aggregated in $\boldsymbol{\theta}=\left[\mathbf{x}^{\mathrm{T}} \boldsymbol{\delta}^{\mathrm{T}} \mathbf{o}^{\mathrm{T}}\right]^{\mathrm{T}}$, where $\mathbf{x}=\left[\mathbf{x}_{1}^{\mathrm{T}}, \ldots, \mathbf{x}_{N}^{\mathrm{T}}\right]^{\mathrm{T}}$, $\mathbf{o}=\left[o_{1}, \ldots, o_{K}\right]^{\mathrm{T}}$, and $\boldsymbol{\delta}=\left[\delta_{L}, \ldots, \delta_{N+M}\right]^{\mathrm{T}}$, in which 
$K=M+N-1$. The communication overhead associated with a localization algorithm (centralized or distributed) is not considered in our analysis. We introduce $\phi_{n m}$ as the angle between nodes $n$ and $m$. To facilitate the scaling analysis, we will assume that, asymptotically in the number of nodes, $\phi_{n m} \sim \mathcal{U}(0,2 \pi), \forall n, m$. This assumption ignores boundary effects of the deployment region, and is thus only valid for agents in the center of the deployment region with favorable geometric conditions.

\section{DELAY-ACCURACY TRADE-OFF DEFINITION}

In this section, we introduce the definition of the delayaccuracy trade-off, denoted by $\delta(\rho)$, defined below, based on the delay and accuracy metrics.

\section{A. Delay Metric: Medium Access Control Delay}

It is supposed that each ranging transmission requires a delay $T=1$ and that transmissions do not overlap in time. If the network operates in CO and NC modes there are $(M+N)$ and $M$ transmissions, respectively. Thus, the total delays are $\mathcal{D}=(M+N) T$ for $\mathrm{CO}$ and $\mathcal{D}=M T$ for $\mathrm{NC}$ mode.

\section{B. Accuracy Metric: Fisher Information}

The Fisher information matrix (FIM) [10] of $\boldsymbol{\theta}$ has form

$$
\mathbf{J}(\boldsymbol{\theta})=\left[\begin{array}{lll}
\boldsymbol{\Phi}(\mathbf{x}, \mathbf{x}) & \boldsymbol{\Phi}(\mathbf{x}, \boldsymbol{\delta}) & \mathbf{\Phi}(\mathbf{x}, \mathbf{o}) \\
\boldsymbol{\Phi}(\boldsymbol{\delta}, \mathbf{x}) & \boldsymbol{\Phi}(\boldsymbol{\delta}, \boldsymbol{\delta}) & \boldsymbol{\Phi}(\boldsymbol{\delta}, \mathbf{o}) \\
\boldsymbol{\Phi}(\mathbf{o}, \mathbf{x}) & \boldsymbol{\Phi}(\mathbf{o}, \boldsymbol{\delta}) & \boldsymbol{\Phi}(\mathbf{o}, \mathbf{o})
\end{array}\right]
$$

in which $\boldsymbol{\Phi}(\mathbf{a}, \mathbf{b})=\mathbb{E}_{\mathbf{z} \mid \boldsymbol{\theta}}\left\{-\nabla_{\mathbf{a}} \nabla_{\mathbf{b}}^{\mathrm{T}} \log p(\mathbf{z} \mid \boldsymbol{\theta})\right\}$. The $\log$ likelihood of $\boldsymbol{\theta}$ is equal to

$$
\log p(\mathbf{z} \mid \boldsymbol{\theta})=-\frac{1}{2 \sigma^{2}} \sum_{i=L}^{N+M} \sum_{j \neq i}\left(z_{i j}-\delta_{i}-d_{i j}-o_{j}\right)^{2} .
$$

The equivalent FIM (EFIM) of $\mathbf{x}$ describes the Fisher information regarding the agents' positions in the absence of knowledge on $\mathbf{o}$ and $\boldsymbol{\delta}$, and is given by

$$
\mathbf{J}^{\mathrm{E}}(\mathbf{x})=\boldsymbol{\Phi}(\mathbf{x}, \mathbf{x})-\mathbf{B C}^{-1} \mathbf{B}^{\mathrm{T}}
$$

in which $\mathbf{B}=[\mathbf{\Phi}(\mathbf{x}, \boldsymbol{\delta}), \boldsymbol{\Phi}(\mathbf{x}, \mathbf{o})]$ and

$$
\mathbf{C}=\left[\begin{array}{ll}
\boldsymbol{\Phi}(\boldsymbol{\delta}, \boldsymbol{\delta}) & \boldsymbol{\Phi}(\boldsymbol{\delta}, \mathbf{o}) \\
\mathbf{\Phi}(\mathbf{o}, \boldsymbol{\delta}) & \boldsymbol{\Phi}(\mathbf{o}, \mathbf{o})
\end{array}\right]
$$

We note that $\mathbf{B C}^{-1} \mathbf{B}^{\mathrm{T}} \succeq 0$, thus reducing the information about the agents' positions, with respect to $\Phi(\mathbf{x}, \mathbf{x})$. The EFIM of a specific agent (say agent $1, \mathbf{J}^{\mathrm{E}}\left(\mathbf{x}_{1}\right)$ ) can similarly be determined [3]. Let the structure of $\mathbf{J}^{\mathrm{E}}(\mathbf{x})$ be in the form of

$$
\mathbf{J}^{\mathrm{E}}(\mathbf{x})=\left[\begin{array}{ll}
\mathbf{J}_{11} & \mathbf{J}_{12} \\
\mathbf{J}_{21}^{\mathrm{T}} & \mathbf{J}_{22}
\end{array}\right],
$$

wherein $\mathbf{J}_{11}$ is $2 \times 2$ matrix corresponding to the information of the position of agent 1 . Then, $\mathbf{J}^{\mathrm{E}}\left(\mathbf{x}_{1}\right)=\mathbf{J}_{11}-\mathbf{J}_{12} \mathbf{J}_{22}^{-1} \mathbf{J}_{21}^{\mathrm{T}}$.

Finally, the positioning accuracy for the network is evaluated using the position error bound (PEB), $\mathcal{P}=$ $\left(\operatorname{tr}\left\{\left[\mathbf{J}^{\mathrm{E}}(\mathbf{x})\right]^{-1}\right\} / N\right)^{1 / 2}$ [3]. The PEB for agent $i$ is equal to $\mathcal{P}_{i}=\left(\operatorname{tr}\left\{\left[\mathbf{J}^{\mathrm{E}}\left(\mathbf{x}_{i}\right)\right]^{-1}\right\}\right)^{1 / 2}$. Assuming that each agent is asymptotically (for large number of nodes) evenly surrounded by the remaining nodes, then $\mathcal{P}=\mathcal{P}_{i}$.

\section{Trade-Off}

Our aim is to evaluate the network performance in terms of positioning accuracy with respect to the total delay required to perform the measurements defined by (1). We focus on the asymptotic behavior of the network, when numbers of agents $N$ and anchors $M$ both increase, with $N=M^{\rho}, \rho>0$. If we express the delay scaling as $\mathcal{D} \in \mathcal{O}\left(f_{\mathcal{D}}(M, \rho)\right)$ and the PEB scaling as $\mathcal{P} \in \mathcal{O}\left(f_{\mathcal{P}}(M, \rho)\right)$, then the delay-accuracy trade-off parameter $\delta(\rho)$ is defined as [9]

$$
\delta(\rho)=-\lim _{M \rightarrow \infty} \frac{\log f_{\mathcal{P}}(M, \rho)}{\log f_{\mathcal{D}}(M, \rho)} .
$$

The larger the value of $\delta(\rho)$, the more favorable trade-off is achieved.

\section{DElay-AcCuracy Trade-OfF Evaluation}

We now determine the value of $\delta(\rho)$ for both cooperative and noncooperative operating modes. Letting $\mathbf{q}_{m n}=$ $\left(\mathbf{x}_{m}-\mathbf{x}_{n}\right) /\left\|\mathbf{x}_{m}-\mathbf{x}_{n}\right\|$ be a unit-length vector pointing from $\mathbf{x}_{n}$ to $\mathbf{x}_{m}$, we will make use of the following result.

Lemma 1. $\quad \sum_{n=1}^{U} \mathbf{q}_{n m} \rightarrow \mathbf{0}, \quad \sum_{n=1}^{U} \mathbf{q}_{n m} \mathbf{q}_{n m}^{\mathrm{T}} \rightarrow \frac{U}{2} \mathbf{I}_{2}$ and $\left(\sum_{n=1}^{U} \mathbf{q}_{n m}\right)\left(\sum_{n=1}^{U} \mathbf{q}_{n m}\right)^{\mathrm{T}} \rightarrow \frac{U}{2} \mathbf{I}_{2}$ as $U \rightarrow \infty$.

Proof. This follows immediately from $\phi_{n m} \sim \mathcal{U}(0,2 \pi)$.

\section{A. Cooperative Operating Mode}

1) Delay scaling: Considering the total delay $\mathcal{D}$, the delay for $\mathrm{CO}$ network scales according to

$$
f_{\mathcal{D}}(M, \rho)= \begin{cases}M^{\rho}, & \text { if } \rho \geq 1 \\ M, & \text { if } \rho<1 .\end{cases}
$$

2) PEB scaling: Individual elements of $\mathbf{J}(\boldsymbol{\theta})$ are summarized in Table I. To evaluate $\mathbf{J}^{\mathrm{E}}(\mathbf{x})$ from (4), we first determine $\mathbf{C}^{-1}$. Using the block matrix inversion formula, it can be shown that $\mathbf{C}^{-1}$ is given by (9), in which $\alpha=\sigma^{2} / K$ and $\beta=\sigma^{2} /\left(K-K^{-1}\right)$. Using (9), $\mathbf{D} \doteq \mathbf{B C}^{-1} \mathbf{B}^{\mathrm{T}}$ is expressed as

$$
\begin{aligned}
\mathbf{D} & =\alpha \boldsymbol{\Phi}(\mathbf{x}, \boldsymbol{\delta}) \boldsymbol{\Phi}(\mathbf{x}, \boldsymbol{\delta})^{\mathrm{T}} \\
& +\alpha^{2} \beta \boldsymbol{\Phi}(\mathbf{x}, \boldsymbol{\delta}) \boldsymbol{\Phi}(\delta, \mathbf{o}) \boldsymbol{\Phi}(\delta, \mathbf{o})^{\mathrm{T}} \boldsymbol{\Phi}(\mathbf{x}, \boldsymbol{\delta})^{\mathrm{T}} \\
& +\alpha^{2} \beta \boldsymbol{\Phi}(\mathbf{x}, \boldsymbol{\delta}) \boldsymbol{\Phi}(\delta, \mathbf{o}) \mathbf{1}_{\mathrm{K}} \boldsymbol{\Phi}(\delta, \mathbf{o})^{\mathrm{T}} \boldsymbol{\Phi}(\mathbf{x}, \boldsymbol{\delta})^{\mathrm{T}} \\
& -\alpha \beta \boldsymbol{\Phi}(\mathbf{x}, \mathbf{o}) \boldsymbol{\Phi}(\delta, \mathbf{o}) \boldsymbol{\Phi}(\mathbf{x}, \boldsymbol{\delta})^{\mathrm{T}} \\
& -\alpha \beta \boldsymbol{\Phi}(\mathbf{x}, \mathbf{o}) \mathbf{1}_{\mathrm{K}} \boldsymbol{\Phi}(\delta, \mathbf{o}) \boldsymbol{\Phi}(\mathbf{x}, \boldsymbol{\delta})^{\mathrm{T}} \\
& -\alpha \beta \boldsymbol{\Phi}(\mathbf{x}, \boldsymbol{\delta}) \boldsymbol{\Phi}(\delta, \mathbf{o}) \boldsymbol{\Phi}(\mathbf{x}, \mathbf{o})^{\mathrm{T}} \\
& -\alpha \beta \boldsymbol{\Phi}(\mathbf{x}, \boldsymbol{\delta}) \boldsymbol{\Phi}(\delta, \mathbf{o}) \mathbf{1}_{\mathrm{K}} \boldsymbol{\Phi}(\mathbf{x}, \mathbf{o})^{\mathrm{T}} \\
& +\beta \boldsymbol{\Phi}(\mathbf{x}, \mathbf{o}) \boldsymbol{\Phi}(\mathbf{x}, \mathbf{o})^{\mathrm{T}}+\beta \boldsymbol{\Phi}(\mathbf{x}, \mathbf{o}) \mathbf{1}_{\mathrm{K}} \boldsymbol{\Phi}(\mathbf{x}, \mathbf{o})^{\mathrm{T}}
\end{aligned}
$$

in which $1_{\mathrm{K}}$ denotes the $K \times K$ unity matrix. Thanks to Lemma 1 , for large $K$, the most dominant components of individual terms in (10) are $\frac{1}{\sigma^{2}} \mathbf{I}_{2 \mathrm{~N}}, \mathbf{0}_{2 \mathrm{~N}}, \frac{2}{\sigma^{2}} \mathbf{I}_{2 \mathrm{~N}}, \mathbf{0}_{2 \mathrm{~N}},-\frac{2}{\sigma^{2}} \mathbf{I}_{2 \mathrm{~N}}$, $\mathbf{0}_{2 \mathrm{~N}},-\frac{2}{\sigma^{2}} \mathbf{I}_{2 \mathrm{~N}}, \frac{1}{\sigma^{2}} \mathbf{I}_{2 \mathrm{~N}}, \frac{2}{\sigma^{2}} \mathbf{I}_{2 \mathrm{~N}}$, respectively, in which $\mathbf{0}_{2 \mathrm{~N}}$ denotes the $2 N \times 2 N$ zero matrix. All the other terms are not significant since they are at maximum proportional to $1 / K^{2}$. Thus, $\mathbf{D} \rightarrow 2 \sigma^{-2} \mathbf{I}_{2 \mathrm{~N}}$ as $K \rightarrow \infty$.

We now show that $\mathbf{J}^{\mathrm{E}}(\mathbf{x}) \rightarrow \mathbf{\Phi}(\mathbf{x}, \mathbf{x})$ when $K \rightarrow \infty$. This follows from the scaling of $\boldsymbol{\Phi}(\mathbf{x}, \mathbf{x})$ : its diagonal submatrices 
TABLE I

FIM ELEMENTS OF (2) FOR THE CO CASE

\begin{tabular}{llll}
\hline $\boldsymbol{\Phi}(\mathbf{a}, \mathbf{b})$ & Dimension & Diagonal entry $[\boldsymbol{\Phi}(\mathbf{a}, \mathbf{b})]_{m m}$ & Off-diagonal entry $[\mathbf{\Phi}(\mathbf{a}, \mathbf{b})]_{m n}$ \\
\hline $\mathbf{\Phi}(\mathbf{x}, \mathbf{x})$ & $2 N \times 2 N$ & $\frac{2}{\sigma^{2}} \sum_{i=1, i \neq m}^{M+N} \mathbf{q}_{m i} \mathbf{q}_{m i}^{\mathrm{T}}$ & $-\frac{2}{\sigma^{2}} \mathbf{q}_{m n} \mathbf{q}_{m n}^{\mathrm{T}}$ \\
$\mathbf{\Phi}(\mathbf{x}, \boldsymbol{\delta})$ & $2 N \times(M+N)$ & $\frac{1}{\sigma^{2}} \sum_{i=1, i \neq m}^{M+N} \mathbf{q}_{m i}$ & $\frac{1}{\sigma^{2}} \mathbf{q}_{m n}$ \\
$\mathbf{\Phi}(\mathbf{x}, \mathbf{o})$ & $2 N \times K$ & $\frac{1}{\sigma^{2}} \sum_{i=1, i \neq m}^{M+N} \mathbf{q}_{m i}$ & $\frac{1}{\sigma^{2}} \mathbf{q}_{m n}$ \\
$\mathbf{\Phi}(\boldsymbol{\delta}, \boldsymbol{\delta})$ & $(M+N) \times(M+N)$ & $\frac{1}{\sigma^{2}} K$ & 0 \\
$\mathbf{\Phi}(\mathbf{o}, \mathbf{o})$ & $K \times K$ & $\frac{1}{\sigma^{2}} K$ & 0 \\
$\mathbf{\Phi}(\boldsymbol{\delta}, \mathbf{o})$ & $(M+N) \times K$ & 0 & $\frac{1}{\sigma^{2}}$ \\
\hline
\end{tabular}

$$
\mathbf{C}^{-1}=\left[\begin{array}{cc}
\alpha \mathbf{I}_{\mathrm{M}+\mathrm{N}}+\alpha^{2} \beta \boldsymbol{\Phi}(\boldsymbol{\delta}, \mathbf{o})\left(\mathbf{I}_{\mathrm{K}}+\mathbf{1}_{\mathrm{K}}\right) \boldsymbol{\Phi}(\boldsymbol{\delta}, \mathbf{o})^{\mathrm{T}} & -\alpha \beta \boldsymbol{\Phi}(\boldsymbol{\delta}, \mathbf{o})\left(\mathbf{I}_{\mathrm{K}}+\mathbf{1}_{\mathrm{K}}\right) \\
-\alpha \beta\left(\mathbf{I}_{\mathrm{K}}+\mathbf{1}_{\mathrm{K}}\right) \boldsymbol{\Phi}(\boldsymbol{\delta}, \mathbf{o})^{\mathrm{T}} & \beta\left(\mathbf{I}_{\mathrm{K}}+\mathbf{1}_{\mathrm{K}}\right)
\end{array}\right]
$$

(see Table I) are $2 \sigma^{-2} \sum_{i=1, i \neq m}^{M+N} \mathbf{q}_{m i} \mathbf{q}_{m i}^{\mathrm{T}} \rightarrow \sigma^{-2} K \mathbf{I}_{2}$ as $K \rightarrow \infty$. A comparison of this scaling of $\boldsymbol{\Phi}(\mathbf{x}, \mathbf{x})$ with respect to the scaling of $\mathbf{D}$ leads to the conclusion that $\mathbf{D}$ is asymptotically negligible. Hence, for networks with many nodes, the uncertainty of the transmission times and offsets does not affect the positioning uncertainty.

Now that we have established the scaling of $\mathbf{J}^{\mathrm{E}}(\mathbf{x})$, we focus on the EFIM of the first agent $\mathbf{J}^{\mathrm{E}}\left(\mathrm{x}_{1}\right)$ and determine its scaling. Due to non-zero off-diagonal elements of $\mathbf{J}^{\mathrm{E}}(\mathbf{x})$, it is difficult to determine the scaling directly. To get a tractable solution, we follow the approach from [3] and determine lower and upper bounds $\mathbf{J}^{\mathrm{L}}\left(\mathbf{x}_{1}\right)$ and $\mathbf{J}^{\mathrm{U}}\left(\mathbf{x}_{1}\right)$ for which $\mathbf{J}^{\mathrm{L}}\left(\mathbf{x}_{1}\right) \preceq$ $\mathbf{J}^{\mathrm{E}}\left(\mathbf{x}_{1}\right) \preceq \mathbf{J}^{\mathrm{U}}\left(\mathbf{x}_{1}\right)$, and then show that these bounds have the same scaling. For $\mathbf{J}^{\mathrm{L}}\left(\mathbf{x}_{1}\right)$, we first reduce the information available in $\mathbf{J}^{\mathrm{E}}(\mathbf{x})$. In particular, we remove the cooperative links between the agents that do not include the first agent. This leads to

$$
\begin{aligned}
& \mathbf{J}^{\mathrm{E}}(\mathbf{x}) \succeq \mathbf{J}^{\mathrm{L}}(\mathbf{x})= \\
& \frac{2}{\sigma^{2}}\left[\begin{array}{cccc}
\sum_{\substack{i=1 \\
i \neq 1}}^{M+N} \mathbf{q}_{1 i} \mathbf{q}_{1 i}^{\mathrm{T}} & -\mathbf{q}_{12} \mathbf{q}_{12}^{\mathrm{T}} & \cdots & -\mathbf{q}_{1 N} \mathbf{q}_{1 N}^{\mathrm{T}} \\
-\mathbf{q}_{21} \mathbf{q}_{21}^{\mathrm{T}} & \sum_{\substack{i=1 \\
i \notin[2, N]}}^{M+N} \mathbf{q}_{2 i} \mathbf{q}_{2 i}^{\mathrm{T}} & \mathbf{0}_{2} & \mathbf{0}_{2} \\
\vdots & \vdots & \ddots & \vdots \\
-\mathbf{q}_{N 1} \mathbf{q}_{N 1}^{\mathrm{T}} & \mathbf{0}_{2} & \mathbf{0}_{2} & \sum_{\substack{i=1 \\
i \notin[2, N]}}^{M+N} \mathbf{q}_{N i} \mathbf{q}_{N i}^{\mathrm{T}}
\end{array}\right]
\end{aligned}
$$

Then, using the Schur complement, the lower bound on EFIM for the first agent reads as

$$
\begin{aligned}
\mathbf{J}^{\mathrm{L}}\left(\mathbf{x}_{1}\right) & =\frac{2}{\sigma^{2}} \sum_{\substack{i=1 \\
i \neq 1}}^{M+N} \mathbf{q}_{1 i} \mathbf{q}_{1 i}^{\mathrm{T}} \\
& -\sum_{i=2}^{N}\left[\mathbf{J}^{\mathrm{L}}(\mathbf{x})\right]_{1 i}\left[\mathbf{J}^{\mathrm{L}}(\mathbf{x})\right]_{i i}^{-1}\left[\mathbf{J}^{\mathrm{L}}(\mathbf{x})\right]_{1 i}^{\mathrm{T}}
\end{aligned}
$$

For sufficiently large $N$ and $M$, the first and second terms tend to $\sigma^{-2} K \mathbf{I}_{2}$ and $2 \sigma^{-2}(N-1)(M+1)^{-1} \mathbf{I}_{2}$, respectively. Thus, the lower bound scales as $\mathbf{J}^{\mathrm{L}}\left(\mathbf{x}_{1}\right) \rightarrow \sigma^{-2} K \mathbf{I}_{2}$. For $\mathbf{J}^{\mathrm{U}}\left(\mathbf{x}_{1}\right)$, we first add additional information to $\mathbf{J}^{\mathrm{E}}(\mathbf{x})$. Particularly, we consider all agents except the first agent to have known positions, thus forcing them to behave exactly as anchors. We obtain $\mathbf{J}^{\mathrm{U}}(\mathbf{x})=\mathbf{J}^{\mathrm{U}}\left(\mathbf{x}_{1}\right)=2 \sigma^{-2} \sum_{i=2}^{M+N} \mathbf{q}_{1 i} \mathbf{q}_{1 i}^{\mathrm{T}} \rightarrow \sigma^{-2} K \mathbf{I}_{2}$. Since EFIM lower $\mathbf{J}^{\mathrm{L}}\left(\mathbf{x}_{1}\right)$ and upper $\mathbf{J}^{\mathrm{U}}\left(\mathbf{x}_{1}\right)$ bounds exhibit the same scaling, we can conclude that the EFIM for the first agent scales as $\mathbf{J}^{\mathrm{E}}\left(\mathbf{x}_{1}\right) \rightarrow \sigma^{-2} K \mathbf{I}_{2}$.

Finally, we determine the PEB scaling, which is $\mathcal{P} \rightarrow$ $\sigma \sqrt{2 / K}$. Therefore

$$
f_{\mathcal{P}}(M, \rho)= \begin{cases}1 / \sqrt{M^{\rho}}, & \text { if } \rho \geq 1, \\ 1 / \sqrt{M}, & \text { if } \rho<1 .\end{cases}
$$

3) Trade-Off: Substitution of (8) and (13) into (7), leads to $\delta(\rho)=\frac{1}{2}, \forall \rho>0$. In other words, in both dense and sparse agent networks, the considered cooperative broadcast ranging strategy offers a fixed trade-off. This trade-off is superior to unicast strategies from [9].

\section{B. Noncooperative Operating Mode}

1) Delay scaling: Considering the total delay $\mathcal{D}$, the delay for NC networks scales according to

$$
f_{\mathcal{D}}(M, \rho)=M, \forall \rho>0 .
$$

2) PEB scaling: Individual elements of $\mathbf{J}(\boldsymbol{\theta})$ are summarized in Table II. The inverse of $\mathbf{C}$ from (5) in the NC mode is lengthier due to a loss of symmetry compared with the $\mathrm{CO}$ mode. This is because not all nodes broadcast in the NC mode. Thus, we evaluate $\mathbf{C}^{-1}$ only asymptotically and we consider $N=M^{\rho}$ in order to address possible different growing rates for the number of agents and anchors. An application of the block matrix inversion formula on (5) filled by sub-matrices from Table II reveals that

$$
\mathbf{C}^{-1} \rightarrow \sigma^{2}\left[\begin{array}{cc}
\frac{1}{M+M^{\rho}} \mathbf{I}_{\mathrm{M}}+\frac{1}{M} \mathbf{1}_{\mathrm{M}} & -\frac{1}{M} \mathbf{1}_{\mathrm{M}, \mathrm{K}} \\
-\frac{1}{M} \mathbf{1}_{\mathrm{M}, \mathrm{K}}^{\mathrm{T}} & \frac{1}{M}\left(\mathbf{I}_{\mathrm{K}}+\mathbf{1}_{\mathrm{K}}\right)
\end{array}\right], \forall \rho>0,
$$

in which $\mathbf{1}_{\mathrm{M}, \mathrm{K}}$ is the $M \times K$ unity matrix. Using this partial result and subsequently analyzing $\mathbf{D}=\mathbf{B C}^{-1} \mathbf{B}^{\mathrm{T}}$, shows that $\mathbf{D} \rightarrow \sigma^{-2} \frac{1}{2} \frac{M^{\rho}+2 M}{M^{\rho}+M} \mathbf{I}_{2 \mathrm{~N}}, \forall \rho>0$. Since $\mathbf{\Phi}(\mathbf{x}, \mathbf{x}) \rightarrow$ $\sigma^{-2}(M / 2) \mathbf{I}_{2 \mathrm{~N}}$, we conclude that, in NC mode, $\mathbf{D}$ is asymptotically negligible $\forall \rho>0$ with respect to $\boldsymbol{\Phi}(\mathbf{x}, \mathbf{x})$ in (4). We can thus consider $\mathbf{J}^{\mathrm{E}}(\mathbf{x}) \rightarrow \mathbf{\Phi}(\mathbf{x}, \mathbf{x})$, which is block-diagonal. Hence, the scaling of EFIM for the first agent $\mathbf{J}^{\mathrm{E}}\left(\mathbf{x}_{1}\right)$ can 
TABLE II

FIM ELEMENTS OF (2) FOR THE NC CASE

\begin{tabular}{|c|c|c|c|}
\hline$\Phi(\mathbf{a}, \mathbf{b})$ & Dimension & Diagonal entry $[\mathbf{\Phi}(\mathbf{a}, \mathbf{b})]_{m m}$ & Off-diagonal entry $[\boldsymbol{\Phi}(\mathbf{a}, \mathbf{b})]_{m n}$ \\
\hline$\Phi(\mathbf{x}, \mathbf{x})$ & $2 N \times 2 N$ & $\frac{1}{\sigma^{2}} \sum_{i=N+1}^{M+N} \mathbf{q}_{m i} \mathbf{q}_{m i}^{\mathrm{T}}$ & 0 \\
\hline$\Phi(\mathbf{x}, \boldsymbol{\delta})$ & $2 N \times M$ & $\frac{\sigma^{2}}{\sigma^{2}} \mathbf{q}_{m(m+N)}$ & $\frac{1}{\sigma^{2}} \mathbf{q}_{m(n+N)}$ \\
\hline$\Phi(\mathbf{x}, \mathbf{o})$ & $2 N \times K$ & $\frac{1}{\sigma^{2}} \sum_{i=N+1}^{M+N} \mathbf{q}_{m i}$ & $\mathbf{0}$ \\
\hline$\Phi(\delta, \delta)$ & $M \times M$ & $\frac{1}{\sigma^{2} K}$ & 0 \\
\hline $\mathbf{\Phi}(\mathbf{o}, \mathbf{o})$ & $K \times K$ & $\begin{cases}\frac{1}{\sigma^{2}} M, & \text { for } m \leq N \\
\frac{1}{\sigma^{2}}(M-1), & \text { otherwise }\end{cases}$ & 0 \\
\hline $\mathbf{\Phi}(\boldsymbol{\delta}, \mathbf{o})$ & $M \times K$ & $\frac{1}{\sigma^{2}}$ & $\begin{cases}0, & \text { for }(m, n) \in \\
\frac{1}{\sigma^{2}}, & \{(i, N+i) \mid i=1, \ldots, M-1\} \\
\text { otherwise }\end{cases}$ \\
\hline
\end{tabular}

be determined easily as $\mathbf{J}^{\mathrm{E}}\left(\mathbf{x}_{1}\right) \rightarrow \sigma^{-2}(M / 2) \mathbf{I}_{2}$. Finally, the PEB scaling is $\mathcal{P} \rightarrow 2 \sigma \sqrt{1 / M}$, so that

$$
f_{\mathcal{P}}(M, \rho)=1 / \sqrt{M}, \forall \rho>0 .
$$

3) Trade-Off: Substituting (14) and (16) into (7), the tradeoff parameter for NC network operating mode is equal to $\delta(\rho)=\frac{1}{2}, \forall \rho>0$. Thus, in both dense and sparse agent networks, the considered noncooperative broadcast ranging strategy offers a fixed trade-off. This trade-off is superior to unicast strategies from [9] and is the same as the cooperative broadcast ranging strategy (from Section IV-A)

\section{Simulation and Discussion}

To understand the behavior of the trade-off parameter as $M \rightarrow+\infty$, we simulated $M \in[10,90]$ anchors and $N=M^{\rho}$ agents, uniformly distributed in a unit-area square environment. We visualize the trade-off for an agent in the center of the environment as well as for all agents. Note that due to boundary effects, the conditions for Lemma 1 will not be valid for agents at the boundary of the environment. Fig. 1 shows the average $\delta$ as a function of $M$ for both operating modes and $\rho \in\{0.5,1.5\}$. For any agent in the center of the environment, the limiting value of $\delta$ is achieved for moderate values of $M$. When also agents in boundary of the environment are considered, the trade-off is $20 \%-30 \%$ below the limiting value, in particular for the NC mode.

Hence, for sufficiently large dense networks and geometrically favorable conditions, $\mathbf{D}=\mathbf{B C}^{-1} \mathbf{B}^{\mathrm{T}}$ is negligible with respect to $\boldsymbol{\Phi}(\mathbf{x}, \mathbf{x})$. This statement holds for both cooperative and noncooperative network operating modes. Therefore, it can be concluded that for a large number of anchors, the lack of synchronization in the network utilizing a broadcast scheme does not compromise the positioning accuracy. It was shown in [9] that for $\rho<1$ the best achievable tradeoff is achieved for TDOA (for which only agents broadcast) with $f_{\mathcal{P}}(M, \rho)=1 / \sqrt{M}$ and $f_{\mathcal{D}}(M, \rho)=M^{\rho}$ and thus $\delta(\rho)=1 /(2 \rho)$. For $\rho>1$, TDOA and unicast strategies leads to a $\lim _{\rho \rightarrow+\infty} \delta(\rho) \rightarrow 0$. To determine the best trade-off for $\rho>1$, we must focus on strategies where agents listen. Since synchronized and non-synchronized networks offer the same PEB scaling, we can limit our search to synchronized networks (i.e., both $\delta$ and $\mathbf{o}$ are known). For such networks, both cooperative and noncooperative modes have fundamentally the

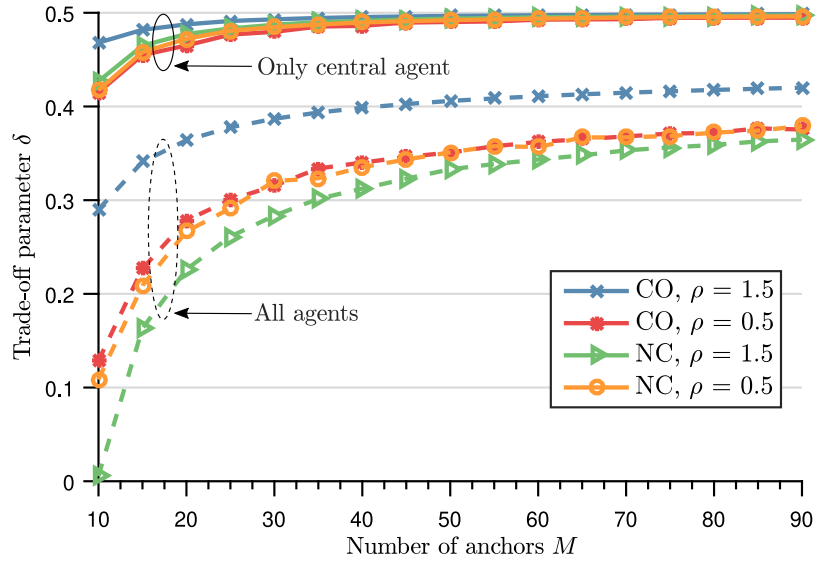

Fig. 1. Simulation of the trade-off parameter as a function of the number of anchors $M$ for a square environment. The solid lines represent the parameter obtained for an agent in the center of the environment, whereas the dashed lines show the parameter evaluated for the overall network.

same behavior: from the point of view of a single agent, they consider all transmitting nodes to be references, each transmission reducing the PEB (proportional to the inverse of the square root of the number of transmissions) and each transmission taking one time resource. Hence, for $\rho>1$, the best possible trade-off is $\delta(\rho)=1 / 2$. Incidentally, this tradeoff was also achieved by unicast strategies from [9] that also allowed for eavesdropping (listening to unicast transmissions) by agents.

\section{CONCLUSION}

We have considered the delay-accuracy trade-off in cooperative and noncooperative networks, performing distance measurements based on broadcasted ranging signals. Due to the nature of these measurements, clock biases and transmission times have been included as unknown parameters. We showed that, even though the presence of these parameters effectively decreases EFIM containing information related to the positioning accuracy, in the asymptotic regime for large number of anchors, this EFIM degradation can be neglected. Hence, the achievable positioning accuracy is the same as in an ideally synchronized network. The trade-off parameter is the same for both cooperative and noncooperative networks, and is equal to one-half. We have also established that for dense agents networks, no better trade-off is achievable. 


\section{REFERENCES}

[1] M. Z. Win, A. Conti, S. Mazuelas, Y. Shen, W. M. Gifford, D. Dardari, and M. Chiani, "Network localization and navigation via cooperation," IEEE Communications Magazine, vol. 49, no. 5, pp. 56-62, may 2011.

[2] N. Patwari, J. N. Ash, S. Kyperountas, A. O. Hero, R. L. Moses, and N. S. Correal, "Locating the nodes: cooperative localization in wireless sensor networks," IEEE Signal Processing Magazine, vol. 22, no. 4, pp. 54-69, 2005.

[3] Y. Shen, H. Wymeersch, and M. Z. Win, "Fundamental Limits of Wideband Localization; Part II: Cooperative Networks," IEEE Transactions on Information Theory, vol. 56, no. 10, pp. 4981-5000, 2010.

[4] F. Sottile, A. Vesco, R. Scopigno, and M. A. Spirito, "MAC layer impact on the performance of real-time cooperative positioning," in 2012 IEEE Wireless Communications and Networking Conference (WCNC), apr 2012, pp. 1858-1863.

[5] C. Lindberg, L. S. Muppirisetty, K. M. Dahln, V. Savic, and H. Wymeersch, "Mac delay in belief consensus for distributed tracking," in 2013 10th Workshop on Positioning, Navigation and Communication (WPNC), March 2013, pp. 1-6.

[6] S. V. de Velde, G. T. F. de Abreu, and H. Steendam, "Improved Censoring and NLOS Avoidance for Wireless Localization in Dense Networks," IEEE Journal on Selected Areas in Communications, vol. 33, no. 11, pp. 2302-2312, nov 2015.

[7] T. Zhang, A. F. Molisch, Y. Shen, Q. Zhang, H. Feng, and M. Z. Win, "Joint Power and Bandwidth Allocation in Wireless Cooperative Localization Networks," IEEE Transactions on Wireless Communications, vol. 15, no. 10, pp. 6527-6540, oct 2016.

[8] L. Song, T. Zhang, X. Yu, C. Qin, and Q. Zhang, "Scheduling in Cooperative UWB Localization Networks Using Round Trip Measurements," IEEE Communications Letters, vol. 20, no. 7, pp. 1409-1412, jul 2016.

[9] G. E. Garcia, L. S. Muppirisetty, E. M. Schiller, and H. Wymeersch, "On the Trade-Off Between Accuracy and Delay in Cooperative UWB Localization: Performance Bounds and Scaling Laws," IEEE Transactions on Wireless Communications, vol. 13, no. 8, pp. 4574-4585, aug 2014.

[10] S. M. Kay, Fundamentals of Statistical Signal Processing: Estimation theory. Prentice-Hall PTR, 1993. 